Review Article

\title{
From Blood to the Brain: Can Systemically Transplanted Mesenchymal Stem Cells Cross the Blood-Brain Barrier?
}

\author{
Linan Liu, ${ }^{1,2}$ Mark A. Eckert, ${ }^{1,2}$ Hamidreza Riazifar, ${ }^{1,2}$ Dong-Ku Kang, ${ }^{1,2}$ \\ Dritan Agalliu, ${ }^{3}$ and Weian Zhao ${ }^{1,2}$ \\ ${ }^{1}$ Department of Pharmaceutical Sciences, Sue and Bill Gross Stem Cell Research Center and Chao Family Comprehensive \\ Cancer Center, University of California, Irvine, 845 Health Sciences Road, Irvine, CA 92697, USA \\ ${ }^{2}$ Department of Biomedical Engineering and Edwards Lifesciences Center for Advanced Cardiovascular Technology, \\ University of California, Irvine, 845 Health Sciences Road, Irvine, CA 92697, USA \\ ${ }^{3}$ Department of Developmental \& Cell Biology, University of California, Irvine, 4236 McGaugh Hall, Irvine, CA 92697, USA
}

Correspondence should be addressed to Weian Zhao; weianz@uci.edu

Received 30 April 2013; Accepted 13 July 2013

Academic Editor: Donald G. Phinney

Copyright (C) 2013 Linan Liu et al. This is an open access article distributed under the Creative Commons Attribution License, which permits unrestricted use, distribution, and reproduction in any medium, provided the original work is properly cited.

Systemically infused mesenchymal stem cells (MSCs) are emerging therapeutics for treating stroke, acute injuries, and inflammatory diseases of the central nervous system (CNS), as well as brain tumors due to their regenerative capacity and ability to secrete trophic, immune modulatory, or other engineered therapeutic factors. It is hypothesized that transplanted MSCs home to and engraft at ischemic and injured sites in the brain in order to exert their therapeutic effects. However, whether MSCs possess the ability to migrate across the blood-brain barrier (BBB) that separates the blood from the brain remains unresolved. This review analyzes recent advances in this area in an attempt to elucidate whether systemically infused MSCs are able to actively transmigrate across the CNS endothelium, particularly under conditions of injury or stroke. Understanding the fate of transplanted MSCs and their CNS trafficking mechanisms will facilitate the development of more effective stem-cell-based therapeutics and drug delivery systems to treat neurological diseases and brain tumors.

\section{Introduction}

Despite enormous advances in our understanding of the molecular and cellular basis of neurological diseases, therapies that lead to sustained improvement or resolution of symptoms have remained elusive. Regenerative therapeutics, that encompass embryonic, neural, and adult stem cell therapies, possess great potential to reverse neuronal damage associated with CNS diseases such as stroke, multiple sclerosis (MS), Parkinson's disease (PD), and Alzheimer's disease (AD) [1]. Mesenchymal stem cells (MSCs) are an especially attractive therapeutic agent due to their ease of isolation, established safety, and potential to target multiple pathways involved in neuronal regeneration. MSCs are connective tissue progenitors that can be readily isolated from multiple tissues including bone marrow and adipose tissue [2-4]. While being initially used for treatment of connective tissue disorders due to their potential to differentiate into bone, cartilage, and fat cells, the discovery that MSCs can secrete cytokines and growth factors with antiapoptotic, proangiogenic, neuroprotective, and immunemodulatory properties has sparked broad clinical interest [24]. In fact, MSCs are the world's first manufactured stem cell product (i.e., Osiris's Prochymal) approved in Canada to treat graft-versus-host disease (GvHD) [5]. MSCs are currently being tested for treating some neurological diseases in multiple ongoing clinical trials, although their exact therapeutic mechanisms in vivo remain largely unknown (i.e., immunomodulation versus secretion of trophic factors that promote tissue regeneration and vascularization) $[1,6,7]$. Furthermore, there is great interest in using MSCs as vehicles to deliver antitumor therapeutics (e.g., tumor necrosis factorrelated apoptosis-inducing ligand (TRAIL), interferon- $\beta$, and oncolytic viruses) for brain tumor treatment [8-10]. 
Given the large number of ongoing clinical trials that use systemic infusion (i.e., intravenously (IV) and intra-arterially (IA)) of MSCs expanded in vitro [2,3], a procedure that is minimally invasive and convenient, it is critical to understand if transplanted MSCs can home to and engraft at ischemic and injured sites in the brain to exert their therapeutic effects. Currently, it is unclear whether systemically infused MSCs can actively migrate across the blood-brain barrier (BBB) that separates the blood and brain. This review attempts to synthesize the recent literature on MSC brain tropism, MSC/BBB interactions, and the underlying molecular mechanisms. We will first briefly introduce how leukocytes and tumor cells transmigrate across the BBB, especially under pathological conditions, to provide a mechanistic framework for the subsequent discussion on MSC homing. We will then concentrate on in vivo and in vitro studies that address whether MSCs actively interact with and transmigrate across the $\mathrm{BBB}$, molecular mechanisms involved in the tropism of MSCs to the injured brain, interactions with the BBB, and biological/therapeutic implications to using MSCs as trophic vehicles for CNS drug delivery. Finally, we will present key challenges and novel approaches that we can utilize in the future in order to effectively study MSC/BBB interactions in vivo and develop MSC-based therapeutics to treat neurological diseases. The study of exogenous MSC homing and distribution into the CNS will not only shed light on how transplanted MSCs exert their therapeutic functions but also will allow us to gain insight into how endogenous MSCs migrate, traffic, and function in response to either CNS injury or other diseases. Additionally, studying MSC trafficking across the $\mathrm{BBB}$ may also contribute to the development of methods to monitor the fate of endogenous and exogenous stem cells in vivo.

\section{Leukocyte Transmigration across the Blood- Brain Barrier (BBB)}

The $\mathrm{BBB}$ is formed by cellular interactions between brain microvascular endothelial cells (BMECs), astrocytes, pericytes, and neurons $[11,12]$. CNS endothelial cells (ECs) exhibit three characteristics that establish their $\mathrm{BBB}$ properties. (a) ECs have TJs that restrict diffusion of ions and polar molecules, resulting in high electrical resistance (TEER) [13, 14]. Endothelial TJs in the CNS are composed of transmembrane proteins Claudins $(-5,-12)$, Occludin, and junctional adhesion molecules (JAMs), as well as cytoplasmic anchoring proteins such as Zonula Occludens proteins (ZO-1, ZO-2). These proteins regulate the paracellular (i.e., between ECs) permeability of endothelial cells $[13,15]$. (b) CNS but not peripheral ECs contain a small number of endocytotic caveolae that serve as intermediates in the receptor-dependent and -independent transcytosis [16]. Caveolae are characterized by expression of Caveolins (Cav-1, -2, and -3), a class of transmembrane proteins $(21-24 \mathrm{kDa})$ that are essential for caveolae formation $[17,18]$. Notably, expression of Cav-1 is upregulated prior to $\mathrm{BBB}$ breakdown following $\mathrm{CNS}$ injury or stroke, concurrent with the increased rate of transcytosis [19, 20]. (c) Finally, CNS endothelium expresses a large number of specific active or passive transporters that regulate passage of nutrients (e.g., glucose or amino acids) from the blood to the brain and prevent drug delivery $[15,21]$.

The BBB plays a vital role in brain homeostasis by restricting the passage of molecules and leukocytes into and out of the brain [22]. However, during brain inflammation and injury, the $\mathrm{BBB}$ becomes compromised and cellular trafficking through the BBB is significantly upregulated [23]. Leukocyte trafficking to sites of CNS inflammation has been well studied and extensively reviewed $[22,24]$. We will only provide a brief overview in order to contrast leukocyte and MSC transmigration across the BBB. Circulating leukocyte transmigration (also called extravasation or diapedesis) through the $\mathrm{BBB}$ occurs primarily at postcapillary venules and is characterized by a multistep adhesion/migration cascade (Figure 1) [25, 26]. During inflammation, BMECs upregulate cell surface adhesion molecules (e.g., P- and Eselectins, vascular cell adhesion molecule-1 (VCAM-1) and Intercellular Adhesion Molecule-1 (ICAM-1)), and chemoattractants (e.g., stromal cell-derived factor-1 (SDF-1) (or CXCL12) and CCL19). Leukocytes initiate transient selectinmediated tethering and rolling on the endothelium that triggers activation of leukocyte integrins such as leukocyte function-associated molecule-1 (LFA-1, ligand for ICAM-1), macrophage-1 antigen (Mac-1, ligand for ICAM-1), and very late antigen-4 (VLA-4, ligand for VCAM-1) and leads to leukocyte arrest on ECs. Leukocytes then undergo actindependent polarization and Mac-1/ICAM-1-mediated lateral "crawling" over the luminal surface. Eventually, leukocytes migrate across the endothelial barrier through both paracellular (i.e., between endothelial cells (ECs)) and transcellular (i.e., directly through individual ECs) pathways, although the transcellular route is preferred [27, 28]. Adhesion of leukocytes on the EC layer induces clustering of endothelial cell surface adhesion molecules (i.e., ICAM-1 and VCAM-1) and triggers downstream signaling pathways that disrupt junctions and promote paracellular migration. Conversely, during transcellular migration, interactions between ICAM-1 and VCAM-1 on the EC surface induce formation of vertical microvilli-like projections (called "transmigratory cups") [27] that provide directional guidance for leukocyte extravasation. Transcellular migration seems to play a major role in leukocyte trafficking in the CNS system where ECs have strong tight junctions [29]. Actin-containing protrusive structures (e.g., podosomes, filopodia, lamellipodia, and pseudopodia) are often formed in leukocytes to enable them to "probe" into, and subsequently penetrate, ECs [27]. In contrast, in some types of CNS injury, activation of ECs and astrocytes can lead to reduced TJ integrity and formation of paracellular gaps, thereby facilitating the migration of leukocytes through a paracellular route. After passing through the endothelial barrier, leukocytes can then penetrate the endothelial basement membrane (BM) and pericytes through gaps within the ECM facilitated by matrix metalloproteinase(MMP-)mediated ECM degradation.

\section{Tropism of MSCs towards Brain}

MSCs delivered systemically have been shown to preferentially localize to sites of inflammation, injury, ischemic 


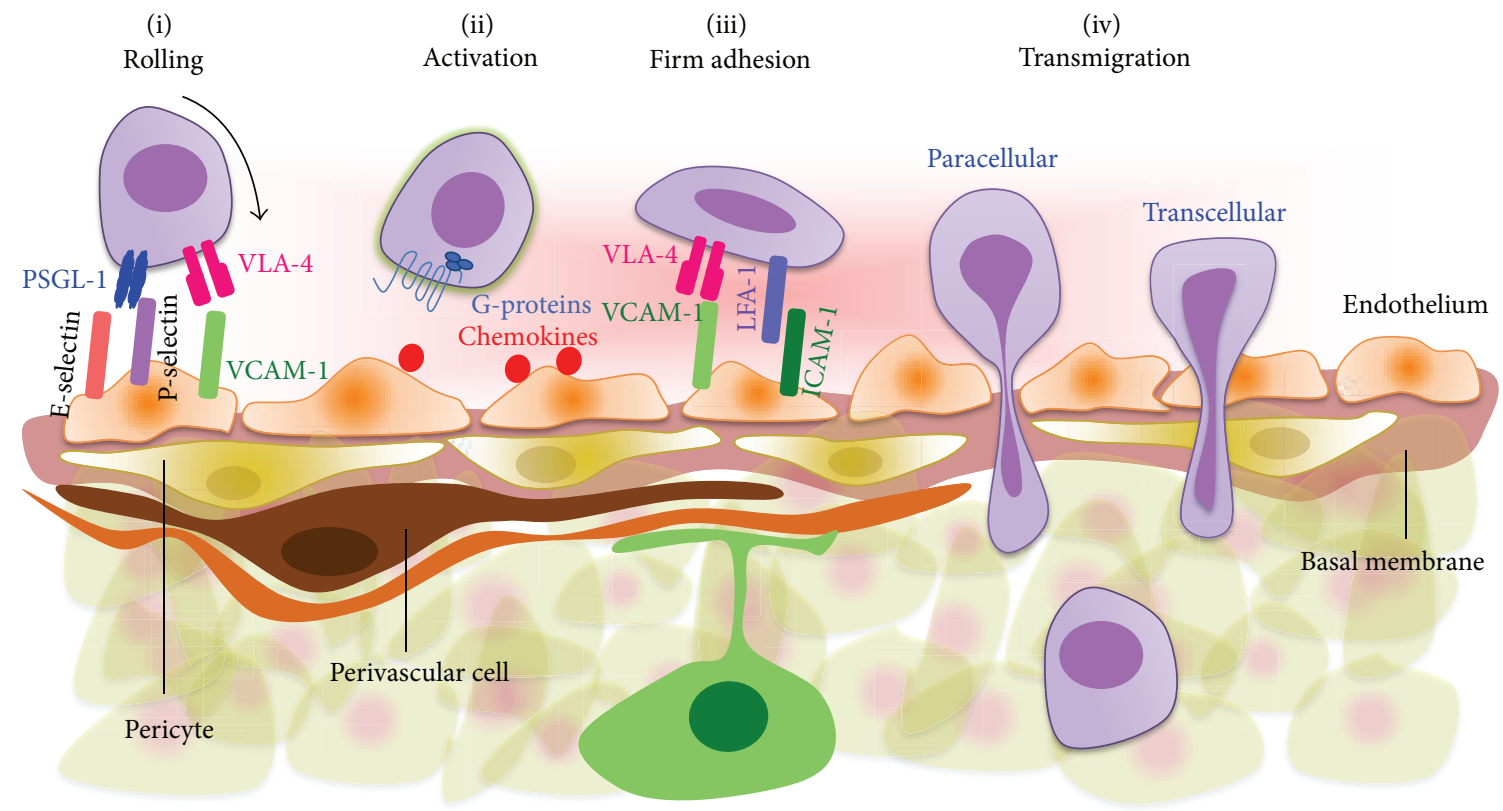

FIGURE 1: Leukocyte extravasation cascade. Leukocytes initially engage with the endothelium via selectin and VCAM-1 mediating interactions during rolling (i), followed by G-protein-mediated activation (ii) and subsequent integrin-mediated firm adhesion (iii). Transmigration across the BBB may occur via paracellular or transcellular routes (iv). It remains to be determined whether systemically infused MSCs possess similar or distinct features and mechanisms enabling them to transmigrate across the BBB and home to the CNS system in vivo.

lesions, and tumors including those in the brain despite their predominant entrapment in the lung vasculature [3,30,31]. For instance, Yilmaz et al. found that intravenously (IV) injected mouse bone-marrow-derived MSCs home to the infarct site in the transient middle cerebral artery occlusion (t-MCAO) model for stroke [31]. The brain tropism for MSCs was confirmed by whole body imaging of radiolabeled MSC given to rats with and without t-MCAO. During the first two hours after stroke, MSCs are transiently trapped in the lungs but migrate over time within the region of brain ischemia [32]. Kim et al. also found that human adipose-derived MSCs (hAMSCs) transplanted through an i.v. route crossed the BBB and migrated into the brain in a mouse model for $\operatorname{AD}[32,33]$. Systemically infused MSCs can also selectively accumulate into certain brain tumors (e.g., gliomas) [8, 10, 34-36]. These studies suggest that MSCs may possess leukocyte-like, active homing mechanisms that enable them to interact with and migrate across the BBB under injury or inflammation. However, the integrity of the cerebral vasculature is likely compromised following injury or inflammation, which can lead to passive MSC accumulation in the brain via entrapment [37]. Therefore, the extent and mechanisms of how MSCs actively cross the BBB remain to be determined.

\section{Molecular Mechanisms of MSC/BBB Interaction and Transmigration}

Several studies have shown that MSCs can utilize a leukocytelike, multistep homing cascade (i.e., rolling, adhesion, and transmigration) to engage with ECs. However, a major caveat of the studies that we will discuss below is the use of cultured EC monolayers including non-BMECs such as human umbilical vein ECs (HUVECs) that do not fully acquire BBB properties typical of the in vivo situation.

MSCs express a variety of leukocyte homing molecules such as chemokine receptors (e.g., CXCR4, CCR2) and cell adhesion molecules (e.g., CD44, integrins $\alpha 4$ and $\beta 1$, and CD99), while they lack some key homing markers including P-selectin glycoprotein ligand 1 (PSGL-1), LFA-1, and Mac-1 [38]. However, studies of MSC-EC interactions and subsequent transmigration have produced conflicting results. Rüster et al. reported that MSCs interact with activated ECs under flow conditions via P-selectin during the initial tethering and rolling steps, although MSCs do not express common P-selectin ligands such as PSGL-1 and CD24 [39]. However, they found that the rolling velocity of MSCs on HUVEC is $100-600 \mu \mathrm{m} / \mathrm{s}$ under shear stress of $0.1-1 \mathrm{dyn} / \mathrm{cm}^{2}$, a value that is significantly higher than that of leukocytes $(\sim 2-$ $100 \mu \mathrm{m} / \mathrm{sec}$ under physiologically relevant shear stress of 1$4 \mathrm{dyn} / \mathrm{cm}^{2}$ ) [39]. On the contrary, several studies reported that MSCs are not able to interact with ECs under flow conditions $[40,41]$. Sackstein et al. showed that native MSCs do not express either PSGL-1 or major functional moieties involved in cell rolling such as sialyl Lewis X (SLeX) and therefore do not bind to P- and E-selectins. MSCs therefore have minimal binding interactions with ECs and they only modestly infiltrate the bone marrow [40]. Similar results were also obtained by Brooke and coworkers [42]. Furthermore, the role of VCAM-1/VLA-4, a receptor/ligand pair that mediates both cell rolling and adhesion, in MSC homing is unclear; few reports [43-45] including that of Rüster et al's [39] stated that VCAM-1/VLA-4 interactions are involved in MSC firm adhesion on ECs and transmigration while others found that MSCs do not bind to VCAM-1 [46]. 
Several studies also investigated MSC transmigration through in vitro endothelial monolayers [40, 45, 47]. In a coculture system of MSC with an endothelial cell monolayer, Steingen and coworkers found that MSCs transmigrated through the endothelial barrier using adhesion molecules including VCAM-1/VLA- 4 and $\beta 1$ integrin [45]. When MSCs were perfused into an isolated heart and investigated using electron microscopy, the authors observed that the tight junctions between endothelial cells became abolished and MSCs interacted with the endothelial cell layer in association with tight cell-cell contacts. In a recent work published by Teo and coworkers, high-resolution confocal and dynamic live-cell imaging has supported an active mode of MSC transmigration across various EC monolayers from lung microvascular endothelial cells (LMVECs) to rat brain ECs (GPNT, a cell line previously used to model the BBB in vitro) [48]. MSCs preferentially transmigrate on $\mathrm{TNF} \alpha$-activated endothelium, rather than naive endothelium, using VCAM-1 and G-protein-coupled receptor signaling- (GPCR-) dependent pathways. MSCs migrate either by paracellular or transcellular diapedesis through discrete gaps or pores in the endothelial monolayer that are enriched for VCAM-1 (transmigratory cups). In contrast to leukocytes, MSC transmigration does not involve significant lateral crawling, presumably due to the lack of Mac-1 expression. Interestingly, MSC exhibited nonapoptotic membrane blebbing activity in the early stages of endothelial transmigration rather than formation of lamellipodia and invadosomes that are normally found in leukocytes, to potentially breach endothelial cells. Finally, MSC transmigration occurred on the time scale of hours. Although the mechanism of MSC transmigration is comparable to leukocyte transmigration across the $\mathrm{BBB}$ in some studies, the time is much longer than leukocyte transmigration in other endothelial systems (usually within minutes) [48]. Yilmaz et al. have studied trafficking of IVinjected mouse bone-marrow-derived MSCs to the brain in the t-MCAO model in vivo and found that interactions between the CD44 on MSCs and P- and E-selectins on ECs mediate MSC recruitment to the CNS [31]. Matsushita et al. have also found that rat MSCs could migrate through a monolayer of rat BMECs in vitro via a paracellular pathway [47] although the underlying mechanism was not reported. Furthermore, Lin et al. recently reported that MSCs trigger tight junction disassembly in human BMEC monolayers through PI3K and ROCK signaling pathways [49].

Similar to immune cells, chemokine receptors and their chemokine ligands are also found to be involved in MSC migration and endothelial transmigration [50-53]. For instance, Chamberlain et al. demonstrated functional expression of various chemokine receptors on murine MSCs using standard Boyden-type chamber assays [50]. More recently, they found that CXCL9, CXCL16, CCL20, and CCL25 were specifically involved in MSC transendothelial migration across murine aortic endothelial cells (MAECs) [41]. In Bloch's studies, they found that cocultivation of MSCs in the presence of bFGF, VEGF, EPO, and IL- 6 resulted in a significant increase of MSC integration with the EC monolayer. They also found that VEGF, EPO, and IL-6 enhanced transmigration, although to different extents, whereas bFGF significantly decreased the transmigration of MSCs [45]. Furthermore, Feng et al. demonstrated that interactions of chemokines and chemokine receptors, specifically through fractalkine-CX3CR1 and SDF-1-CXCR4, partly mediated the migration of rat MSCs to the impaired site in the brain after hypoglossal nerve injury [54].

Finally, the activation of MMPs is also found to be associated with MSC transendothelial migration via degradation of the endothelial BM in vitro, providing a potential mechanism for MSC homing and extravasation into injured tissues in vivo [55]. MSCs constitutively express MMP-2 and membrane type $1 \mathrm{MMP}$ (MT1-MMP) that may play a role in MSC invasion in reconstituted BM matrigel. In particular, Becker et al. [55] found that MSC transmigration across in vitro bone marrow endothelium is at least partially regulated by MMP-2. Interestingly, they also demonstrated that high culture confluence of MSCs was found to increase production of the endogenous MMP-inhibitor TIMP-3 and decrease transendothelial migration of MSCs. The involvement of MMPs in MSC transmigration is also supported by Bloch's study where MSCs-derived MMP-2 but not MMP-9 is found at sites of BM invasion and degradation [45]. Interestingly, TIMP3 expressed by IV administered MSCs is a key player in ameliorating BBB permeability in rodent models after traumatic brain injury (TBI) by blocking vascular endothelial growth factor-A-induced breakdown of endothelial cell adherens junctions [56]. These findings elucidate a potential molecular mechanism for the beneficial effects of MSCs in treating neurological diseases through regulation of $\mathrm{BBB}$ integrity.

\section{MSC as a Delivery Vehicle for Brain Tumors}

The fact that the BBB restricts the passage of molecules of molecular weight $>400$ Dalton presents a great challenge in delivering therapeutics to treat brain tumors and certain CNS diseases. Besides their endogenous therapeutic effects, the tropic properties of MSCs provide unique opportunities to use them as vehicles for gene and drug delivery to treat brain tumors. For instance, Nakamizo and coworkers have demonstrated that MSCs were capable of migrating into glioma xenografts in vivo after intravascular or local delivery [35]. They also found that MSCs engineered to produce IFN- $\beta$ significantly increased animal survival compared with controls in a U87 intracranial glioma xenograft mouse model [35]. Recently, Kim et al. have tested combination therapy for malignant glioma with TRAIL-secreting MSCs and the lipoxygenase inhibitor MK886 that can increase sensitivity to TRAIL-induced apoptosis [8]. They found that MSCbased TRAIL gene delivery combined with MK886 had greater therapeutic efficacy than single-agent treatment in an orthotopic glioma xenografted mouse model [8]. Interestingly, MSCs can also be used as target-delivery vehicle for anticancer drug-loaded biodegradable nanoparticles [57]. This approach may be advantageous over genetic modification with respect to safety and controlled drug release. Roger et al. have found that coumarin- 6 containing polylactic acid nanoparticles and lipid nanocapsules can be efficiently internalized into MSCs without affecting cell viability 
or differentiation [36]. Furthermore, they reported that nanoparticle-loaded cells were able to migrate toward an experimental human glioma model, suggesting that MSCs can serve as cellular carriers for drug-loaded nanoparticles to treat brain tumors.

\section{Conclusion and Perspectives}

MSC transplantation via systemic administration holds enormous potential to treat numerous neurological and brain diseases. However, the in vivo efficacy of MSC therapy has not been well established, and some recent clinical trials have produced mixed results $[2,3]$. The lack of efficacy is attributed largely to an incomplete understanding of MSC biology and their fate following transplantation in vivo $[2,3]$. In particular, crossing the BBB may be a prerequisite for MSCs to exert their therapeutic effects in treating neurological diseases or CNS injury $[3,30,31]$ and is necessary for their use as vehicles for drug delivery to treat brain tumors [58]. It seems clear that, at least in vitro, MSCs possess leukocyte-like, although inefficient, molecular mechanisms involving adhesion molecules, chemokines, and proteases which enable MSC/EC interactions and transmigration. The large discrepancies between studies may be due to the inherent heterogeneity of MSCs combined with variations in experimental techniques and models. A major caveat of in vitro studies is the use of EC monolayers that do not fully recapitulate the in vivo $\mathrm{BBB}$ properties. It will be important to incorporate other BBB cell types, such as primary astrocytes, pericytes, reconstituted basement membrane, and relevant dynamic flow conditions in order to develop more robust in vitro systems for studying MSC/EC interactions. Despite the in vitro evidence, it remains elusive whether systemically infused MSCs are able to use leukocyte-like homing cascades to actively interact with and transmigrate across the BBB in vivo under both normal and pathological conditions. Indeed, it is not clear if MSCs are actually able to actively home or rather are passively "captured" at sites of inflamed and disrupted vessels. Physical factors may act in concert with active homing mechanisms to stop or slow down cells before adhesion interactions subsequently arrest MSCs on ECs.

In order to fully understand the dynamic behavior of transplanted MSCs, imaging of transplanted cells in both the brain and other tissues is required. Both short- and long-term monitoring of cell fate in vivo have benefited from improved molecular imaging techniques to visualize cell survival, biodistribution, and behavior [59-62]. Magnetic resonance-based tracking of transplanted cells has confirmed that MSCs rapidly localize to infracted regions of the brain [63-65]. Alternatively, a powerful approach for understanding transplanted cell behavior at the single-cell level is to utilize intravital imaging techniques to study MSC/BBB interactions. In particular, novel transgenic models where TJs between endothelial cells of the BBB or endothelial caveolae are fluorescently tagged may illuminate the mode and dynamics of MSC transmigration in the brain and elsewhere [59]. The study of exogenous MSC homing mechanisms in vivo will not only shed light on how transplanted MSCs exert their therapeutic functions in treating neurological diseases but also will allow us to gain insight into how endogenous MSCs migrate, traffic, and function in response to injury. The mechanistic study of MSC tropism to the brain will also facilitate development of MSCs that are engineered with key homing molecules through genetic or chemical modifications in order to improve MSC targeting and drug delivery in case their basal homing process is inefficient [40,60]. Finally, the elucidation of stem cell fate following transplantation that is believed to be a major bottleneck in stem cell therapy will have broad implications in understanding stem cell functions and developing more effective stem-cell-based therapeutics $[2,3]$.

\section{Acknowledgments}

This work is supported by the start-up fund from the Department of Pharmaceutical Sciences, Sue and Bill Gross Stem Cell Research Center and the Chao Family Comprehensive Cancer Center at UC Irvine, and NCI Cancer Center Support Grant 5P30CA062203-18. M. A. Eckert is supported by a California Institute for Regenerative Medicine (CIRM) Training Grant (TG2-01152).

\section{References}

[1] A. Trounson, "New perspectives in human stem cell therapeutic research," BMC Medicine, vol. 7, article 29, 2009.

[2] J. Ankrum and J. M. Karp, "Mesenchymal stem cell therapy: two steps forward, one step back," Trends in Molecular Medicine, vol. 16, no. 5, pp. 203-209, 2010.

[3] J. M. Karp and G. S. L. Teo, "Mesenchymal stem cell homing: the devil is in the details," Cell Stem Cell, vol. 4, no. 3, pp. 206-216, 2009.

[4] A. I. Caplan and D. Correa, “The MSC: an injury drugstore," Cell Stem Cell, vol. 9, no. 1, pp. 11-15, 2011.

[5] D. Cyranoski, "Canada approves stem cell product," Nature Biotechnology, vol. 30, no. 7, p. 571, 2012.

[6] G. Brooke, M. Cook, C. Blair et al., "Therapeutic applications of mesenchymal stromal cells," Seminars in Cell and Developmental Biology, vol. 18, no. 6, pp. 846-858, 2007.

[7] P. Dharmasaroja, "Bone marrow-derived mesenchymal stem cells for the treatment of ischemic stroke," Journal of Clinical Neuroscience, vol. 16, no. 1, pp. 12-20, 2009.

[8] S. M. Kim, J. S. Woo, C. H. Jeong, C. H. Ryu, J. Y. Lim, and S. S. Jeun, "Effective combination therapy for malignant glioma with TRAIL-secreting mesenchymal stem cells and lipoxygenase inhibitor MK886," Cancer Research, vol. 72, no. 18, pp. 48074817, 2012.

[9] M. Studeny, F. C. Marini, J. L. Dembinski et al., "Mesenchymal stem cells: potential precursors for tumor stroma and targeteddelivery vehicles for anticancer agents," Journal of the National Cancer Institute, vol. 96, no. 21, pp. 1593-1603, 2004.

[10] A. U. Ahmed, M. A. Tyler, B. Thaci et al., "A comparative study of neural and mesenchymal stem cell-based carriers for oncolytic adenovirus in a model of malignant glioma," Molecular Pharmaceutics, vol. 8, no. 5, pp. 1559-1572, 2011.

[11] N. J. Abbott, A. A. K. Patabendige, D. E. M. Dolman, S. R. Yusof, and D. J. Begley, "Structure and function of the blood-brain barrier," Neurobiology of Disease, vol. 37, no. 1, pp. 13-25, 2010. 
[12] B. V. Zlokovic, "The blood-brain barrier in health and chronic neurodegenerative disorders," Neuron, vol. 57, no. 2, pp. 178-201, 2008.

[13] J. M. Anderson and C. M. van Itallie, "Physiology and function of the tight junction," Cold Spring Harbor Perspectives in Biology, vol. 1, no. 2, Article ID a002584, 2009.

[14] L. L. Rubin and J. M. Staddon, "The cell biology of the bloodbrain barrier," Annual Review of Neuroscience, vol. 22, pp. 11-28, 1999.

[15] R. Daneman, L. Zhou, D. Agalliu, J. D. Cahoy, A. Kaushal, and B. A. Barres, "The mouse blood-brain barrier transcriptome: a new resource for understanding the development and function of brain endothelial cells," PLoS ONE, vol. 5, no. 10, Article ID e13741, 2010.

[16] S. A. Predescu, D. N. Predescu, and A. B. Malik, "Molecular determinants of endothelial transcytosis and their role in endothelial permeability," The American Journal of PhysiologyLung Cellular and Molecular Physiology, vol. 293, no. 4, pp. L823-L842, 2007.

[17] M. Drab, P. Verkade, M. Elger et al., "Loss of caveolae, vascular dysfunction, and pulmonary defects in caveolin-1 genedisrupted mice," Science, vol. 293, no. 5539, pp. 2449-2452, 2001.

[18] M. P. Lisanti, Z. Tang, and M. Sargiacomo, "Caveolin forms a hetero-oligomeric protein complex that interacts with an apical GPI-linked protein: Implications for the biogenesis of caveolae," Journal of Cell Biology, vol. 123, no. 3, pp. 595-604, 1993.

[19] C. Kaur and E. A. Ling, "Blood brain barrier in hypoxicischemic conditions," Current Neurovascular Research, vol. 5, no. 1, pp. 71-81, 2008.

[20] S. Nag, J. L. Manias, and D. J. Stewart, "Expression of endothelial phosphorylated caveolin-1 is increased in brain injury," Neuropathology and Applied Neurobiology, vol. 35, no. 4, pp. 417426, 2009.

[21] J. A. Nicolazzo and K. Katneni, "Drug transport across the blood-brain barrier and the impact of breast cancer resistance protein (ABCG2)," Current Topics in Medicinal Chemistry, vol. 9, no. 2, pp. 130-147, 2009.

[22] R. M. Ransohoff and B. Engelhardt, "The anatomical and cellular basis of immune surveillance in the central nervous system," Nature Reviews Immunology, vol. 12, no. 9, pp. 623-635, 2012.

[23] C. Uboldi, A. Döring, C. Alt, P. Estess, M. Siegelman, and B. Engelhardt, "L-Selectin-deficient SJL and C57BL/6 mice are not resistant to experimental autoimmune encephalomyelitis," European Journal of Immunology, vol. 38, no. 8, pp. 2156-2167, 2008.

[24] B. Engelhardt, "Immune cell entry into the central nervous system: involvement of adhesion molecules and chemokines," Journal of the Neurological Sciences, vol. 274, no. 1-2, pp. 23-26, 2008.

[25] E. H. Wilson, W. Weninger, and C. A. Hunter, "Trafficking of immune cells in the central nervous system," Journal of Clinical Investigation, vol. 120, no. 5, pp. 1368-1379, 2010.

[26] B. Engelhardt and R. M. Ransohoff, "The ins and outs of T-lymphocyte trafficking to the CNS: anatomical sites and molecular mechanisms," Trends in Immunology, vol. 26, no. 9, pp. 485-495, 2005.

[27] C. V. Carman, "Mechanisms for transcellular diapedesis: probing and pathfinding by 'invadosome-like protrusions,"' Journal of Cell Science, vol. 122, part 17, pp. 3025-3035, 2009.
[28] K. Ley, C. Laudanna, M. I. Cybulsky, and S. Nourshargh, "Getting to the site of inflammation: the leukocyte adhesion cascade updated," Nature Reviews Immunology, vol. 7, no. 9, pp. 678-689, 2007.

[29] M. von Wedel-Parlow, S. Schrot, J. Lemmen, L. Treeratanapiboon, J. Wegener, and H. Galla, "Neutrophils cross the BBB primarily on transcellular pathways: an in vitro study," Brain Research, vol. 1367, pp. 62-76, 2011.

[30] A. R. Simard and S. Rivest, "Bone marrow stem cells have the ability to populate the entire central nervous system into fully differentiated parenchymal microglia," FASEB Journal, vol. 18, no. 9, pp. 998-1000, 2004.

[31] G. Yilmaz, S. Vital, C. E. Yilmaz, K. Y. Stokes, J. S. Alexander, and D. N. Granger, "Selectin-mediated recruitment of bone marrow stromal cells in the postischemic cerebral microvasculature," Stroke, vol. 42, no. 3, pp. 806-811, 2011.

[32] S. Kim, K. A. Chang, J. Kim et al., "The preventive and therapeutic effects of intravenous human adipose-derived stem cells in Alzheimer's disease mice," PLoS ONE, vol. 7, no. 9, Article ID e45757, 2012.

[33] D. Jeon, K. Chu, S. Lee et al., "A cell-free extract from human adipose stem cells protects mice against epilepsy," Epilepsia, vol. 52, no. 9, pp. 1617-1626, 2011.

[34] C. Pendleton, Q. Li, D. A. Chesler, K. Yuan, H. GuerreroCazares, and A. Quinones-Hinojosa, "Mesenchymal stem cells derived from adipose tissue versus bone marrow: in vitro comparison of their tropism towards gliomas," PLoS ONE, vol. 8, no. 3, Article ID e58198, 2013.

[35] A. Nakamizo, F. Marini, T. Amano et al., "Human bone marrowderived mesenchymal stem cells in the treatment of gliomas," Cancer Research, vol. 65, no. 8, pp. 3307-3318, 2005.

[36] M. Roger, A. Clavreul, M. Venier-Julienne et al., "Mesenchymal stem cells as cellular vehicles for delivery of nanoparticles to brain tumors," Biomaterials, vol. 31, no. 32, pp. 8393-8401, 2010.

[37] R. H. Lee, A. A. Pulin, M. J. Seo et al., "Intravenous hMSCs improve myocardial infarction in mice because cells embolized in lung are activated to secrete the anti-inflammatory protein TSG-6," Cell Stem Cell, vol. 5, no. 1, pp. 54-63, 2009.

[38] L. D. S. Meirelles, A. I. Caplan, and N. B. Nardi, "In search of the in vivo identity of mesenchymal stem cells," Stem Cells, vol. 26, no. 9, pp. 2287-2299, 2008.

[39] B. Rüster, S. Göttig, R. J. Ludwig et al., "Mesenchymal stem cells display coordinated rolling and adhesion behavior on endothelial cells," Blood, vol. 108, no. 12, pp. 3938-3944, 2006.

[40] R. Sackstein, J. S. Merzaban, D. W. Cain et al., "Ex vivo glycan engineering of CD44 programs human multipotent mesenchymal stromal cell trafficking to bone," Nature Medicine, vol. 14, no. 2, pp. 181-187, 2008.

[41] G. Chamberlain, H. Smith, G. E. Rainger, and J. Middleton, "Mesenchymal stem cells exhibit firm adhesion, crawling, spreading and transmigration across aortic endothelial cells: effects of chemokines and shear," PLoS ONE, vol. 6, no. 9, Article ID e25663, 2011.

[42] G. Brooke, H. Tong, J. Levesque, and K. Atkinson, "Molecular trafficking mechanisms of multipotent mesenchymal stem cells derived from human bone marrow and placenta," Stem Cells and Development, vol. 17, no. 5, pp. 929-940, 2008.

[43] J. E. Ip, Y. Wu, J. Huang, L. Zhang, R. E. Pratt, and V. J. Dzau, "Mesenchymal stem cells use integrin $\beta 1$ not CXC chemokine receptor 4 for myocardial migration and engraftment," Molecular Biology of the Cell, vol. 18, no. 8, pp. 2873-2882, 2007. 
[44] V. F. M. Segers, I. van Riet, L. J. Andries et al., "Mesenchymal stem cell adhesion to cardiac microvascular endothelium: activators and mechanisms," American Journal of PhysiologyHeart and Circulatory Physiology, vol. 290, no. 4, pp. H1370H1377, 2006.

[45] C. Steingen, F. Brenig, L. Baumgartner, J. Schmidt, A. Schmidt, and W. Bloch, "Characterization of key mechanisms in transmigration and invasion of mesenchymal stem cells," Journal of Molecular and Cellular Cardiology, vol. 44, no. 6, pp. 1072-1084, 2008.

[46] A. Heiskanen, T. Hirvonen, H. Salo et al., "Glycomics of bone marrow-derived mesenchymal stem cells can be used to evaluate their cellular differentiation stage," Glycoconjugate Journal, vol. 26, no. 3, pp. 367-384, 2009.

[47] T. Matsushita, T. Kibayashi, T. Katayama et al., "Mesenchymal stem cells transmigrate across brain microvascular endothelial cell monolayers through transiently formed inter-endothelial gaps," Neuroscience Letters, vol. 502, no. 1, pp. 41-45, 2011.

[48] G. S. Teo, J. A. Ankrum, R. Martinelli et al., "Mesenchymal stem cells transmigrate between and directly through tumor necrosis factor- $\alpha$-activated endothelial cells via both leukocyte-like and novel mechanisms," Stem Cells, vol. 30, no. 11, pp. 2472-2486, 2012.

[49] M. N. Lin, D. S. Shang, W. Sun et al., "Involvement of PI3K and ROCK signaling pathways in migration of bone marrow-derived mesenchymal stem cells through human brain microvascular endothelial cell monolayers," Brain Research, vol. 1513, pp. 1-8, 2013.

[50] G. Chamberlain, K. Wright, A. Rot, B. Ashton, and J. Middleton, "Murine mesenchymal stem cells exhibit a restricted repertoire of functional chemokine receptors: comparison with human," PLoS ONE, vol. 3, no. 8, Article ID e2934, 2008.

[51] V. Sordi, M. L. Malosio, F. Marchesi et al., "Bone marrow mesenchymal stem cells express a restricted set of functionally active chemokine receptors capable of promoting migration to pancreatic islets," Blood, vol. 106, no. 2, pp. 419-427, 2005.

[52] M. Honczarenko, Y. Le, M. Swierkowski, I. Ghiran, A. M. Glodek, and L. E. Silberstein, "Human bone marrow stromal cells express a distinct set of biologically functional chemokine receptors," Stem Cells, vol. 24, no. 4, pp. 1030-1041, 2006.

[53] A. L. Ponte, E. Marais, N. Gallay et al., "The in vitro migration capacity of human bone marrow mesenchymal stem cells: comparison of chemokine and growth factor chemotactic activities," Stem Cells, vol. 25, no. 7, pp. 1737-1745, 2007.

[54] J. Feng, J. B. P. He, S. T. Dheen, and S. S. W. Tay, "Interactions of chemokines and chemokine receptors mediate the migration of mesenchymal stem cells to the impaired site in the brain after hypoglossal nerve injury," Stem Cells, vol. 22, no. 3, pp. 415-427, 2004.

[55] A. de Becker, P. van Hummelen, M. Bakkus et al., "Migration of culture-expanded human mesenchymal stem cells through bone marrow endothelium is regulated by matrix metalloproteinase- 2 and tissue inhibitor of metalloproteinase3," Haematologica, vol. 92, no. 4, pp. 440-449, 2007.

[56] T. Menge, Y. Zhao, J. Zhao et al., "Mesenchymal stem cells regulate blood-brain barrier integrity through TIMP3 release after traumatic brain injury," Science Translational Medicine, vol. 4, no. 161, Article ID 161ra150, 2012.

[57] Z. Gao, L. Zhang, J. Hu, and Y. Sun, "Mesenchymal stem cells: a potential targeted-delivery vehicle for anti-cancer drug, loaded nanoparticles," Nanomedicine, vol. 9, no. 2, pp. 174-184, 2013.
[58] A. H. Klopp, A. Gupta, E. Spaeth, M. Andreeff, and F. Marini III, "Concise review: dissecting a discrepancy in the literature: do mesenchymal stem cells support or suppress tumor growth?" Stem Cells, vol. 29, no. 1, pp. 11-19, 2011.

[59] D. Agalliu and I. Schieren, "Heterogeneity in the developmental potential of motor neuron progenitors revealed by clonal analysis of single cells in vitro," Neural Development, vol. 4, no. 1, article 2, 2009.

[60] D. Sarkar, J. A. Spencer, J. A. Phillips et al., "Engineered cell homing," Blood, vol. 118, no. 25, pp. e184-e191, 2011.

[61] B. J. Schaller, J. F. Cornelius, N. Sandu, and M. Buchfelder, "Molecular imaging of brain tumors personal experience and review of the literature," Current Molecular Medicine, vol. 8, no. 8, pp. 711-726, 2008.

[62] N. Sandu, T. Spiriev, and B. Schaller, "Stem cell transplantation in neuroscience: the role of molecular imaging," Stem Cell Reviews, vol. 8, no. 4, pp. 1265-1266, 2012.

[63] W. C. Shyu, C. P. Chen, S. Z. Lin, Y. J. Lee, and H. Li, "Efficient tracking of non-iron-labeled mesenchymal stem cells with serial MRI in chronic stroke rats," Stroke, vol. 38, no. 2, pp. 367374, 2007.

[64] O. Detante, S. Valable, F. de Fraipont et al., "Magnetic resonance imaging and fluorescence labeling of clinical-grade mesenchymal stem cells without impacting their phenotype: study in a rat model of stroke," Stem Cells Translational Medicine, vol. 1, no. 4, pp. 333-340, 2012.

[65] S. S. Lu, S. Liu, Q. Q. Zu et al., "In vivo MR imaging of intraarterially delivered magnetically labeled mesenchymal stem cells in a canine stroke model," PLoS ONE, vol. 8, no. 2, Article ID e54963, 2013. 

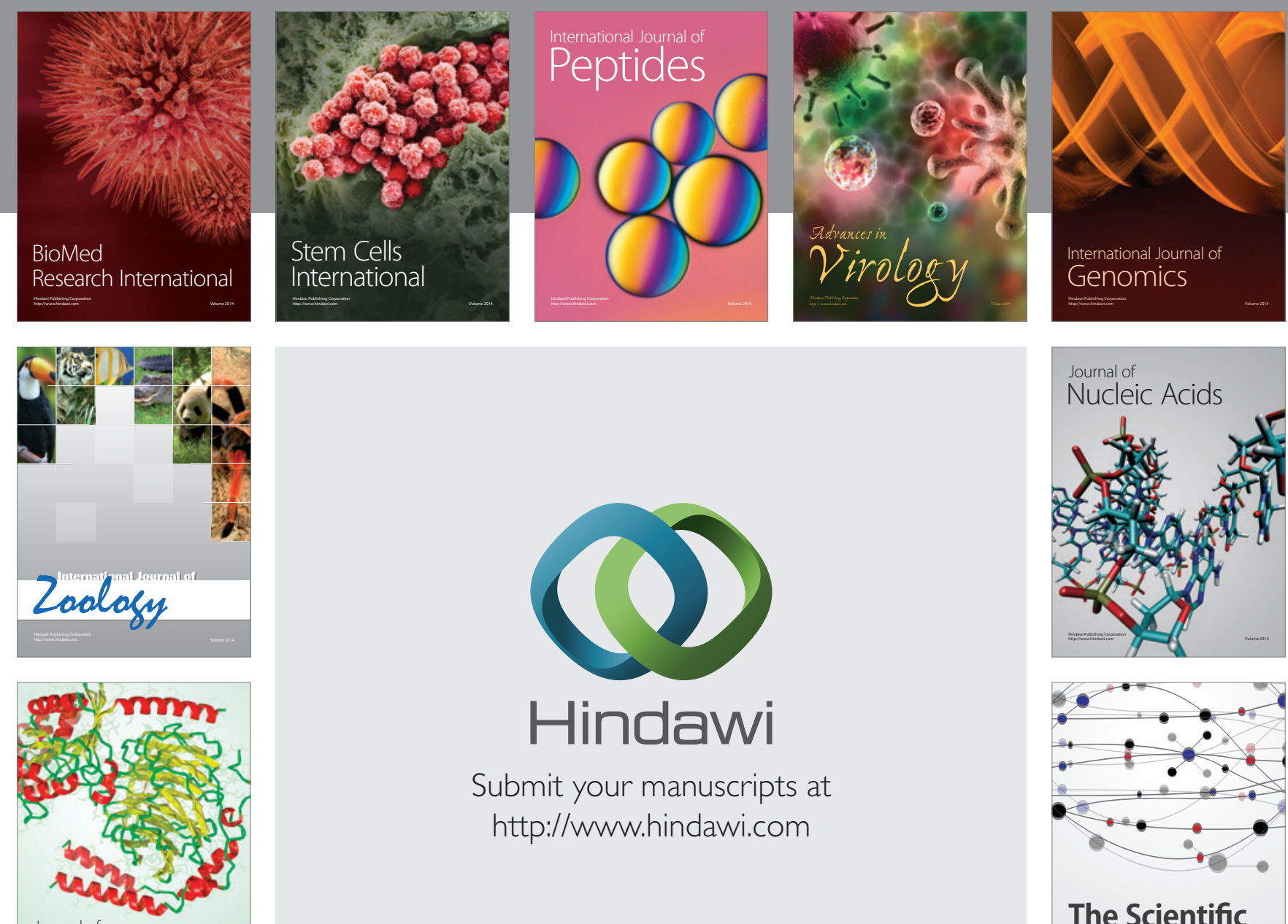

Submit your manuscripts at

http://www.hindawi.com

Journal of
Signal Transduction
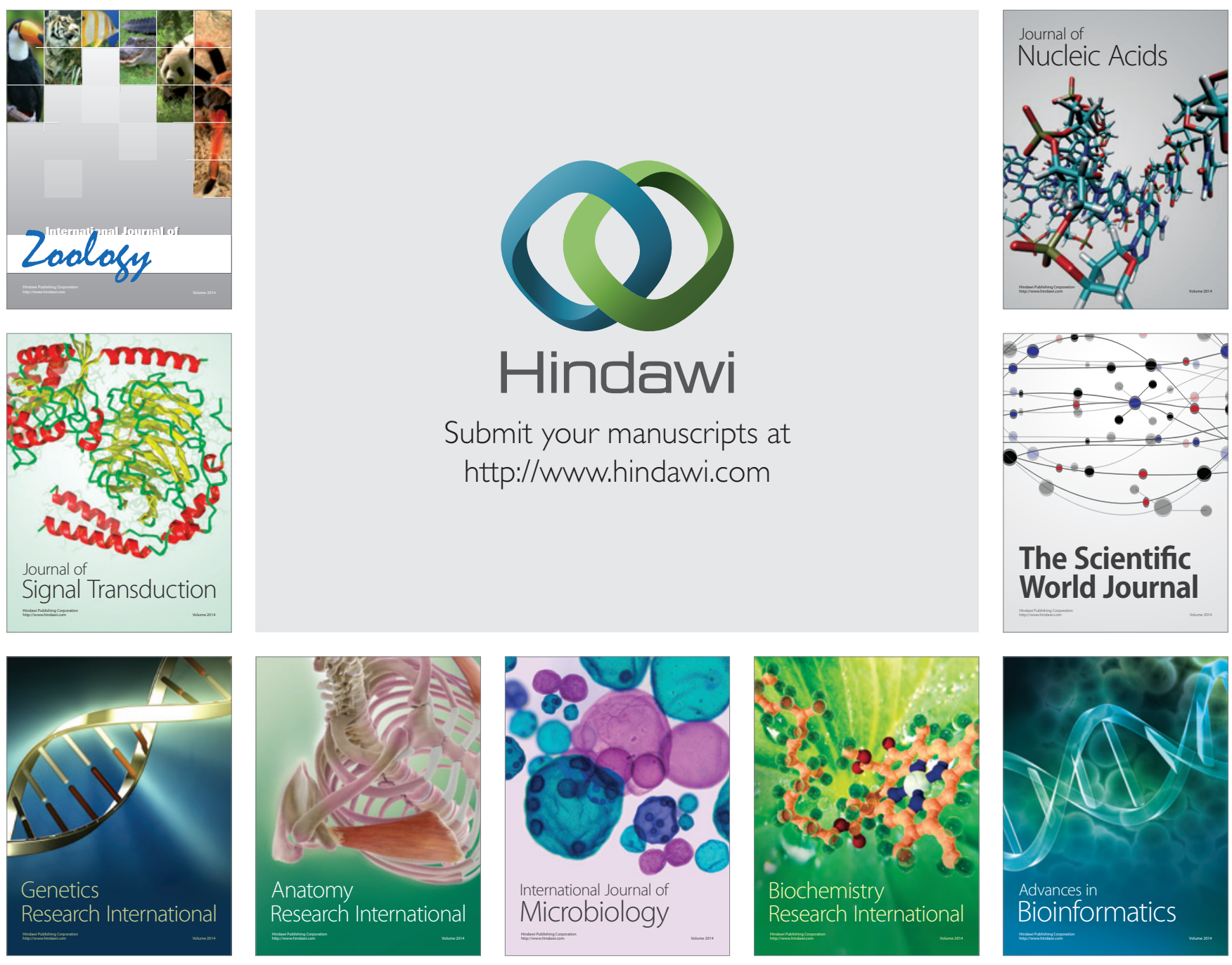

The Scientific World Journal
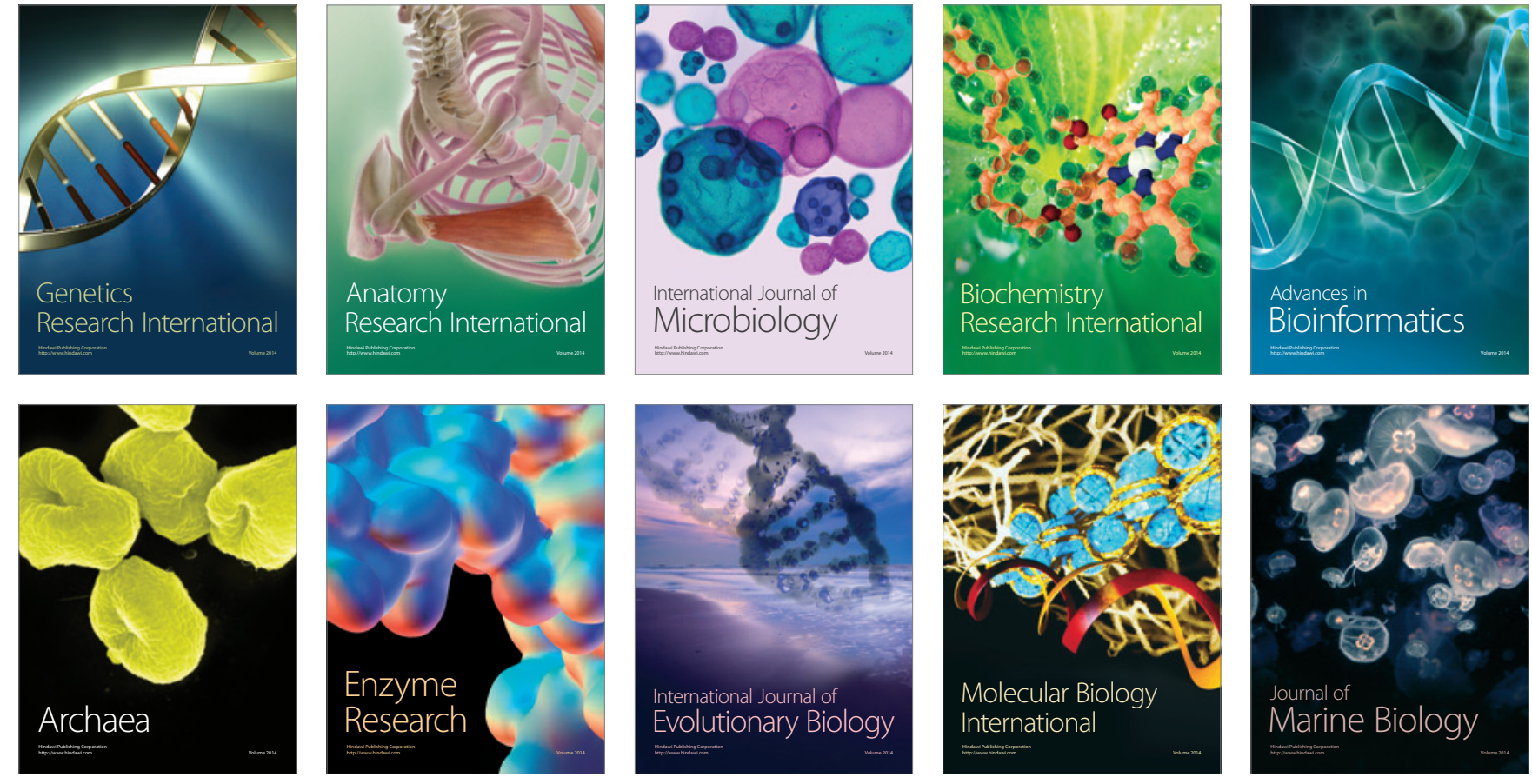